\title{
The problem of flow by-pass at permeable reactive barriers
}

\author{
H. Klammler ${ }^{1,2,3}$ \& K. Hatfield ${ }^{1,2}$ \\ ${ }^{1}$ Dept. of Civil and Coastal Engineering, Univ. of Florida, USA \\ ${ }^{2}$ Inter-Disciplinary Program in Hydrologic Sciences, \\ Univ. of Florida, USA \\ ${ }^{3}$ Dept. of Hydraulic Engineering and Water Resources Management, \\ Graz Univ. of Technology, Austria
}

\begin{abstract}
Permeable Reactive Barriers (PRBs) are a passive in-situ technology, which is based on the interception and physical, chemical and/or biological remediation of a contaminant plume through installation of reactive material in an aquifer. An analytical approach in two dimensions has been introduced that allows for the determination of the flow fields and capture zones near PRBs of different types. The present work uses this approach to investigate the problem of flow by-pass, which is when a portion of flow both enters and leaves the PRB through the upgradient side of the reactor without crossing it. This occurs when a significant ambient flow component exists parallel to the PRB and may lead to contaminant flow divergence around the PRB. Maximum permissible deviations of the ambient groundwater flow direction are defined for a range of PRB types in order to avoid by-pass. Results show for rectangular continuous wall reactors, deviations in the design ambient groundwater flow direction become even more important as the reactor becomes more elongated perpendicular to that direction. This undermines the typical engineering assumption that a PRB longer than the transverse width of the plume is sufficient for plume intercept and treatment. The addition of perpendicular funnel arms or velocity equalization walls at the reactor acts favorably against flow by-pass. For drain and gate PRBs, susceptibility to flow by-pass may be reduced by increasing the separation of collector and distribution drains. An increase in the hydraulic resistance of the reactor increases the possibility of flow by-pass.
\end{abstract}

Keywords: groundwater, contamination, plume, remediation, capture zone, conformal mapping. 


\section{Introduction}

The presence and transport of contaminant plumes in the groundwater is a more than widely recognized threat to the human health and environment. Permeable reactive barriers (PRBs) represent a passive and in-situ alternative to pump-andtreat systems that require a continuous energy supply and commonly include extraction of contaminated groundwater to the surface for treatment (Cunningham and Reinhard [1]). The working principle of PRBs is based on the installation of a reactive medium in the flow path of a contaminant plume, which is thus forced by the natural groundwater gradient to migrate through the reactive medium in the subsurface. Physical, chemical and/or biological processes between the reactive medium and the contaminants lead to the desired degradation and/or deposition of the contaminants as they cross the reactive cell, thus performing the actual remediation process before the groundwater reenters the natural aquifer (Environmental Protection Agency [2]). While a given reactor cell may or not possess impermeable side walls, the treated portion of the groundwater flow can be increased by deploying impermeable cutoff walls extending from the reactor sides into the aquifer, which is referred to as the funnel-and-gate (F\&G; Starr and Cherry [3]) system. Velocity equalization walls (VEWs; Painter [4]) have been proposed to achieve a more uniform flux distribution entering the reactor. Furthermore, draining trenches can be used in the drain-and-gate (D\&G; Bundesministerium für Bildung und Forschung [5]) configuration, which act as collectors of contaminated groundwater up-gradient of the reactor and as distributors of clean water on the down-gradient side. An example of each of these PRB types is depicted in fig. 1.

A fundamental issue for the design and operation of a PRB is the hydraulics of the groundwater flow, since it determines both size and location of the capture zone. The problem of flow by-pass as defined by Craig et al. [6] is concerned with deviations of the ambient groundwater flow direction from the general design direction perpendicular to a PRB and the resulting effect that some of the captured flow may leave the reactor through the up-gradient side of the PRB without crossing the reactor. This may lead to contaminant divergence around a PRB and, hence, non-compliance with remediation goals. Furthermore, flow bypass is accompanied by the effect that some flow enters the reactor through the down-gradient side of the PRB. The shaded zones in figs. 1(a) and 1(b) illustrate situations where flow by-pass occurs, while figs. 1(c) through 1(e) represent cases where is does not. Fig. 1(a) corresponds to the particular case of a simple CW PRB, where flow can enter and leave through the side walls (see related discussion about major and minor capture widths in [6] and Klammler and Hatfield [7]). The flow fields of fig. 1 are based on analytical solutions presented by Klammler and Hatfield [7, 8], who make use of a two-dimensional conformal mapping/superposition approach and who give a more detailed review of literature related to the topic. The goal of the present study is to apply their results in order to determine the maximum deviation of the ambient groundwater flow direction before flow by-pass occurs, in dependence of (1) PRB type and dimensions and (2) hydraulic resistance of the reactor. For this 

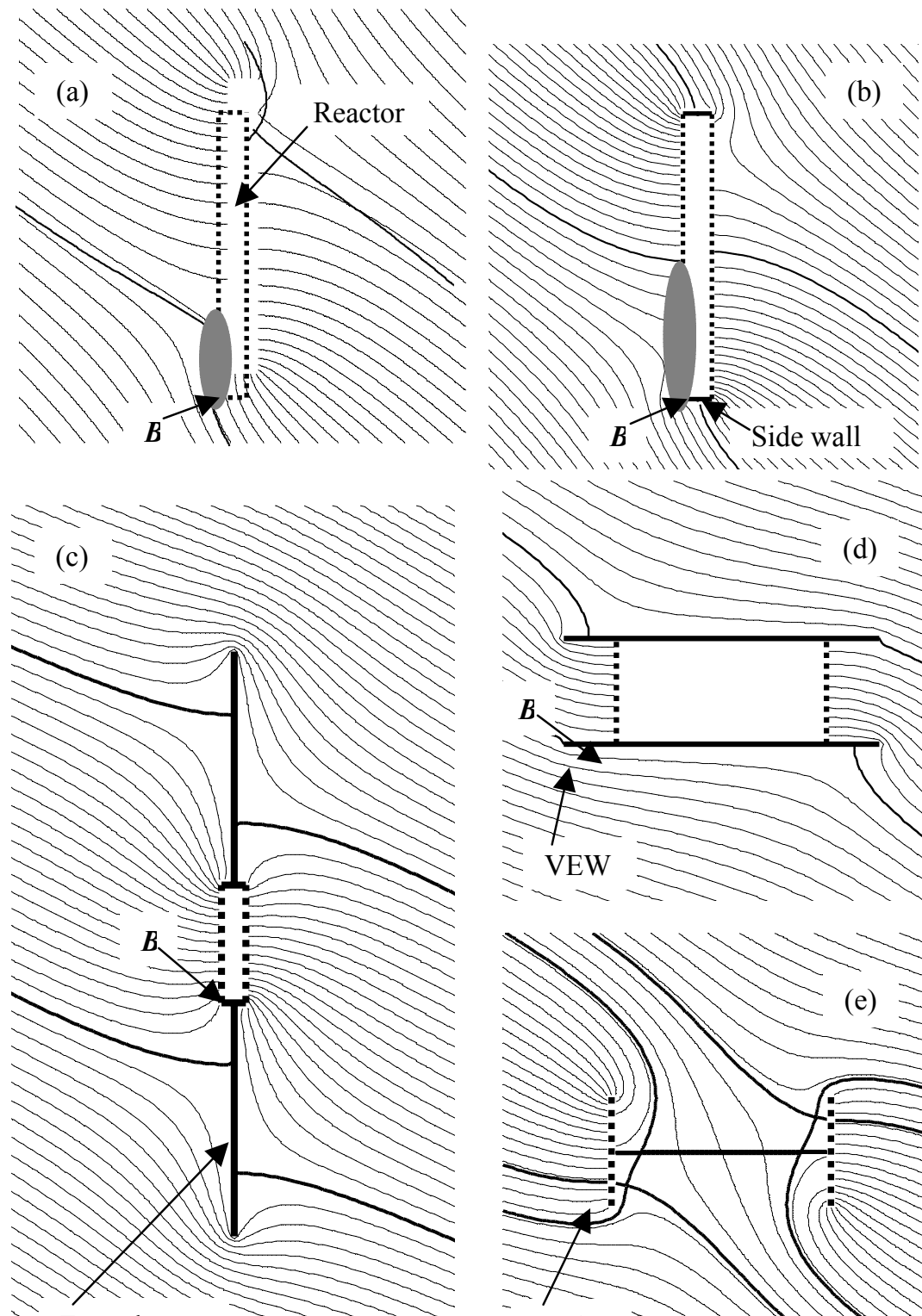

Funnel arm
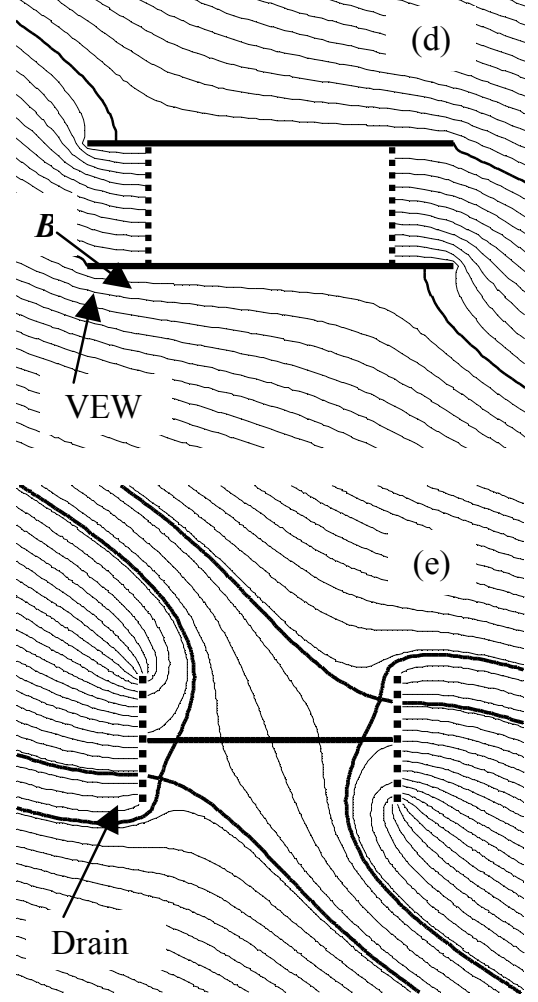

Figure 1: Configurations and flow fields of different PRB types. (a) simple CW, (b) CW with impermeable side walls, (c) F\&G, (d) PRB with VEW and (e) D\&G. Thick solid lines represent impermeable boundaries, thick dotted lines constant head boundaries and thick stream lines delimit capture zones. Shaded ellipses in (a) and (b) indicate flow by-pass. 
purpose, the mathematical condition for flow by-pass is formulated below and respective results are represented graphically for illustration, validation and discussion.

\section{Theory}

In fig. 1 the design groundwater flow direction is assumed from left to right (positive $x$-direction) corresponding to an angle $\alpha=90^{\circ}$. The actual groundwater flow directions in fig. 1 are chosen as $\alpha<90^{\circ}$, i.e., from the top left to the bottom right. It is easily recognized that the following discussion may be restricted to the range $0^{\circ} \leq \alpha \leq 90^{\circ}$, since flow fields for $90^{\circ}<\alpha<360^{\circ}$ can be obtained from double symmetry of the flow domains. Intuitively, the limiting state of flow by-pass may be defined as the situation when the local flux at the bottom left corner of the reactor is zero, i.e., when flow neither enters nor leaves the reactor at this location (stagnation point). Denoting the respective groundwater flow direction at this limiting state with $\alpha_{f}, \alpha<\alpha_{f}$ indicates flow by-pass, since then local fluxes exist at the up-gradient face of the reactor which are directed outwards into the aquifer (figs. 1(a),(b). Mathematically, this means that for $\alpha=\alpha_{f}$

$$
\frac{q_{x B}}{q_{y B}}=1
$$

where $q_{x B}$ and $q_{y B}\left[\mathrm{~L}^{2} / \mathrm{T}\right]$ are the magnitudes of depth integrated local fluxes due to ambient flow components $q_{x}$ and $q_{y}\left[\mathrm{~L}^{2} / \mathrm{T}\right]$ in the $x$ and $y$-directions, respectively, at point $B$ being the bottom left corner of the reactor. According to potential theory, $q_{x B}$ and $q_{y B}$ are determined from taking derivatives of the solutions for the complex potentials of the respective case given in [7,8].

Maintaining the notation for conformal mapping planes from [7, 8], it is recognized that eqn (1) may be directly applied in the $\tau_{l}$-plane containing a PRB mapped onto the real axis of the half-plane rather than the physical plane containing the actual PRB. This is due to the fact that both $q_{x B}$ and $q_{y B}$ are scaled by the same factor when mapped from the physical onto the half-plane. Hence, from [7, 8], for simple CW PRBs (fig. 1(a)) $q_{x B}$ may be found as

$$
q_{x B}=\left.q_{x} \frac{d \tau_{2}}{d \tau_{1}}\right|_{\tau_{1}=\tau_{1 B}}=q_{x}\left|\frac{k \tau_{1 B}}{\sqrt{1-k^{2} \tau_{1 B}^{2}}}\right|
$$

where $\tau_{l B}=( \pm) 1$ is the location of $B$ in the $\tau_{1}$-plane and the absolute value of the derivative is taken to obtain $q_{x B}$ as a pure magnitude. $k$ [-] here is a parameter from 0 to 1 and determined by the shape (aspect ratio) of the PRB as presented by $[7,8]$. For flow in the $y$-direction $q_{y B}=q_{y}$ is immediately obtained and knowing that $\tan (\alpha)=q_{x} / q_{y}$ eqn (1) leads to

$$
\alpha_{f}=\arctan \frac{\sqrt{1-k^{2}}}{k}
$$

which is represented by the solid line in fig. 2 . 
For PRBs with impermeable side walls, VEW or of the F\&G type (figs. 1(b),(c),(d)) results of [8] are again used to express

$$
q_{x B}=\left.q_{x} \frac{d \tau_{5}}{d \tau_{1}}\right|_{\tau_{1}=\tau_{1 B}}=q_{x}\left|\frac{k\left(e^{2}-\tau_{1 B}^{2}\right)}{\sqrt{\left(1-\tau_{1 B}^{2}\right)\left(1-k^{2} \tau_{1 B}^{2}\right)}}\right| .
$$

Besides the aspect ratio of the reactor, PRB shape parameter $k$ includes the possible presence and dimensions of the VEW or funnel arms. $e^{2}[-]$ is a hydraulic properties parameter of the PRB accounting for the conductivity ratio between aquifer and reactor material and ranges from 0.5 to 1 [8]. $q_{y B}$ is found as

$$
q_{y B}=\left.q_{y} \frac{d \tau_{3}}{d \tau_{1}}\right|_{\tau_{1}=\tau_{1 B}}=q_{y}\left|\frac{\tau_{1 B}}{\sqrt{\left(1-\tau_{1 B}^{2}\right)}}\right|
$$

and knowing $\tau_{1 B}=( \pm) 1$ eqn (1) gives

$$
\alpha_{f}=\arctan \frac{\sqrt{1-k^{2}}}{k\left(1-e^{2}\right)}
$$

which is represented by the thick solid contour lines of $\alpha_{f}$ in fig. 3 .

In the case of a D\&G PRB (fig. 1(e)), it is not obvious at what location flow by-pass will first occur, i.e., at what location flow will first start to leave the upgradient face of the reactor (collector drain). In other words, although flow bypass may be expected to start on the right side of the bottom half of the collector drain, the exact location $B$ is not known a priori. However, eqn (4) is still valid and knowing $q_{y B}=q_{y}$ it is the case that eqn (1) leads to

$$
\tan \alpha=\frac{\sqrt{\left(1-\tau_{1 B}^{2}\right)\left(1-k^{2} \tau_{1 B}^{2}\right)}}{k\left(e^{2}-\tau_{1 B}^{2}\right)} .
$$

This can be viewed as a quadratic equation in $\tau_{1 B}{ }^{2} ; \tau_{1 B}{ }^{2}$ because there are symmetric points on the up and down-gradient drains where eqn (1) holds, which correspond to $q_{x B}$ and $q_{y B}$ being equal and having opposite (stagnation point) or equal directions. If $\tau_{1 B}{ }^{2}$ possesses complex roots, then no stagnation point is present on the collector drain, i.e., flow enters the collector drain everywhere. If $\tau_{l B}{ }^{2}$ possesses a pair of real roots, then stagnation points are present on the collector drain indicating flow by-pass. The limiting case, hence, where flow bypass starts, is when the roots of $\tau_{l B}{ }^{2}$ are identical, i.e., the discriminant of eqn (7) is 0 . Imposing this condition finally gives

$$
\alpha_{f}=\arctan \frac{1-k^{2}}{2 k \sqrt{\left(1-e^{2}\right)\left(1-e^{2} k^{2}\right)}}
$$

which is represented by the thin solid contour lines of $\alpha_{f}$ in fig. 3 .

\section{Results and discussion}

Figs. 2 and 3 graphically represent results from eqns (3), (6) and (8) and allow for easy determination of the angle $\alpha_{f}$ at which flow by-pass first occurs. The dimensionless parameters $k$ and $e$ facilitate an efficient and closed form 
representation of results for $\alpha_{f}$ and are given by [7, 8] for the range of PRB types discussed. $k$ is a parameter related to the geometric shape of the PRB structure, while $e$ contains information about the hydraulic properties of the PRB. Obviously, the absolute size of the PRB and the magnitude of the ambient groundwater flow are not relevant for the problem at hand. The solid line in fig. 2 illustrates the dependence of $\alpha_{f}$ on $k^{2}$ for simple CW PRBs (fig. 1(a); eqn (3)) without significant hydraulic losses in the reactor (i.e., infinite conductivity of reactive material). Since $k$ increases with the aspect ratio (length in $y$-direction over width in $x$-direction) of the reactor, it may be seen how $\alpha_{f}$ increases towards $90^{\circ}$ as the reactor becomes longer in the direction perpendicular to the design groundwater flow direction. That is, for simple CW PRBs, long and narrow designs are susceptible to flow by-pass at smaller deviations of the ambient groundwater flow direction from $\alpha=90^{\circ}$. The dashed line in fig. 2 corresponds to CW PRBs with impermeable side walls (fig. 1(b)) and negligible hydraulic losses in the reactor. The respective relationship is described by eqn (6), where $e$ for each $k$ is determined from [8] for a hydraulic resistance $R=0[-]$ of the reactor. Since $k$ is the same for simple CW PRBs and $\mathrm{CW}$ PRBs with impermeable side walls of equal aspect ratio, fig. 2 indicates a stronger susceptibility to flow by-pass in the presence of impermeable side walls. This may be attributed to the fact that simple CW PRBs allow flow through the sides of the reactor, which is not considered as flow by-pass according to the present definition. In this context it is required to recall the discussion about major and minor capture widths for simple CW PRBs as given in $[6,7]$.

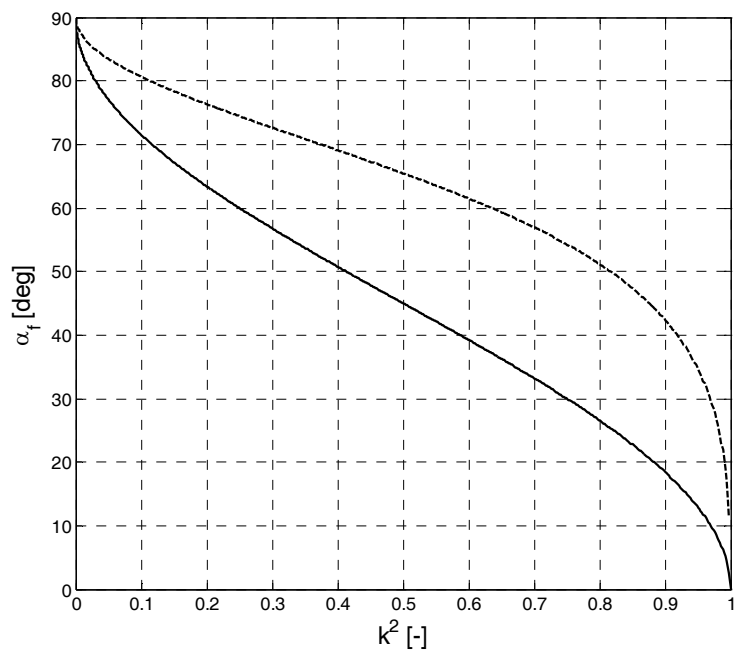

Figure 2: $\quad \alpha_{f}$ for simple CW PRBs (solid) and for CW PRBs with impermeable side walls (dashed).

Fig. 3 shows $\alpha_{f}$ in dependence of $k^{2}$ and $e^{2}$ for the PRB configurations of figs. 1(b) through 1(e). The dark solid lines correspond to eqn (6), which is valid 
for PRBs with impermeable side walls or VEW as well as for F\&G PRBs. $k$ is known to increase as VEW or funnel arms become longer, which translates into smaller values of $\alpha_{f}$ and a larger tolerance to fluctuations in the groundwater flow direction before flow by-pass occurs. Also, $e$ is known to increase with $R$ showing that flow by-pass starts earlier (i.e., at larger $\alpha_{f}$ ) as the hydraulic losses in the reactor are larger. The dashed line in the bottom represents minimum values of $e$ for given $k$ corresponding to zero hydraulic losses in the reactor $(R=$ $0)$. Above the dashed line $e$ increases with $R$ until $e=1$, which is the point where flow divergence [8] begins. Flow divergence refers to the effect of flow by-pass on both sides of the reactor for $\alpha=90^{\circ}$ due to an elevated hydraulic resistance of the reactor. Accordingly, for $e=1$ (as also for $k=0$ ) $\alpha_{f}=90^{\circ}$ in eqns (6), (8) and fig. 3. The light solid lines correspond to the D\&G configuration and eqn (8). Here, $k$ is known to increase with the ratio between drain separation distance and drain length indicating that $\alpha_{f}$ may be limited by a sufficient drain separation distance. $e$ again increases with $R$ indicating, as for all previous cases, a larger hydraulic reactor resistance increases the susceptibility to flow by-pass. Fig. 3 demonstrates that, for a given combination of $k$ and $e, \mathrm{D} \& \mathrm{G}$ PRBs possess a lower $\alpha_{f}$ than PRBs with impermeable side structures. However, for a wide range of typical PRB field configurations D\&G PRBs have rather small values of $k$ and large values of $e$ when compared to F\&G PRBs or PRBs with VEWs. This fact invalidates a direct comparison between flow by-pass for D\&G and other PRB types discussed here.

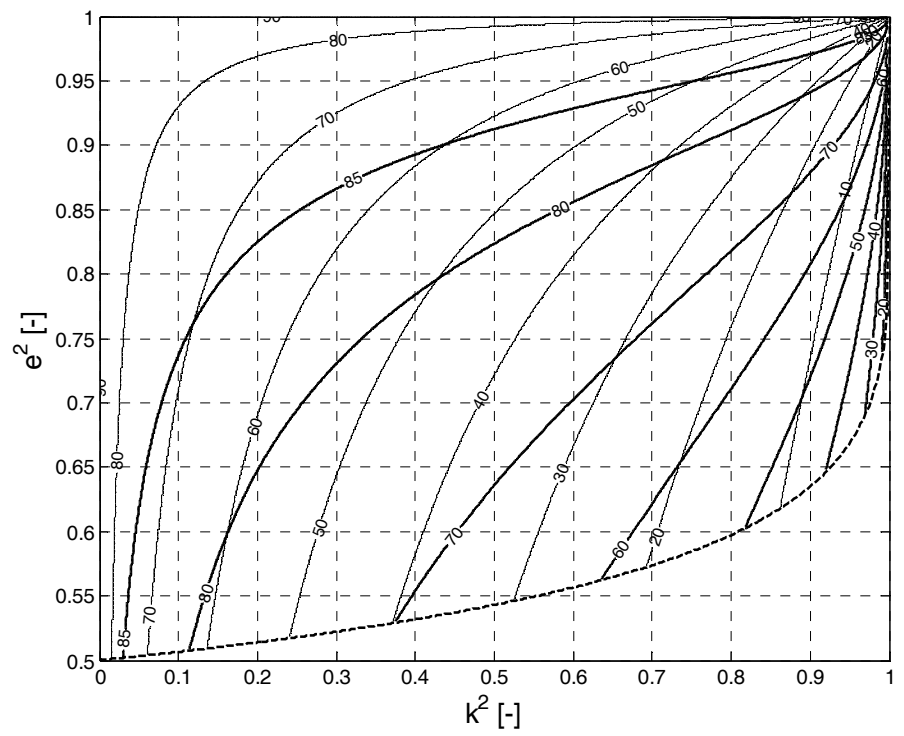

Figure 3: $\quad \alpha_{f}[\mathrm{deg}]$ as a function of $k^{2}$ and $e^{2}$ for PRBs with impermeable side walls, VEW or F\&G PRBs (thick solid) as well as D\&G PRBs (thin solid). Dashed line represents minimum of $e^{2}$ for given $k^{2}$ (no hydraulic losses). 

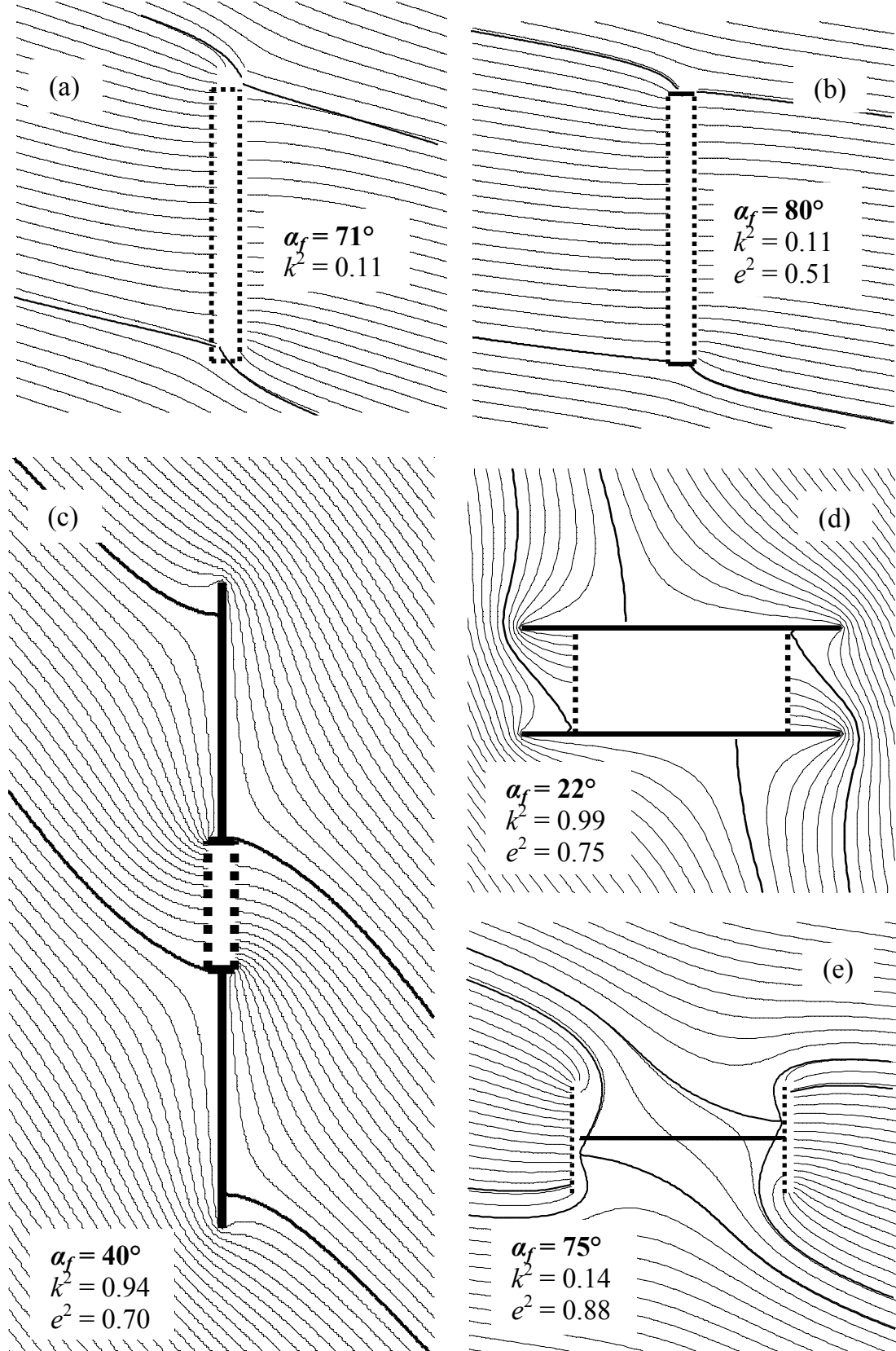

Figure 4: $\quad$ Flow fields and parameters for the examples of fig. 1 for the limiting cases of transition to flow by-pass.

Fig. 4 finally illustrates an example with respective parameters of every PRB type introduced in fig. 1 at the limiting state of $\alpha=\alpha_{f}$, where flow by-pass starts. 
The purpose is to validate the presented results and to give an idea of the range of values of $\alpha_{f}, k^{2}$ and $e^{2}$ to expect in practice. The thick stream lines delimiting the capture zones are seen to meet the PRB at the bottom left corner of the reactor or, in the case of $D \& G P R B$, at the single stagnation point on the collector drain. Any further deviation of the groundwater flow direction from its design direction would lead to a certain amount of flow by-pass in each case. While the geometric parameters used in fig. 4 are apparent, the hydraulic parameters were chosen as follows: $(a, b)$ infinite reactor conductivity; $(c)$ equal reactor and aquifer conductivities; (d) reactive material 10 times more conductive than aquifer; (e) squared reactor of 1/10 of aquifer thickness and reactive material 100 times more conductive than aquifer.

\section{Summary}

Taking advantage of analytical solutions for flow fields near different types of PRBs presented by $[7,8]$ this study focuses on the problem of flow by-pass. In particular, the angles of the ambient groundwater flow direction are determined for incipient flow by-pass, i.e., at which flow starts leaving through the upgradient face of the reactor. Results for simple $\mathrm{CW}, \mathrm{CW}$ with impermeable side walls, F\&G, VEW and D\&G PRBs are represented in closed form analytical expressions as well as graphically. Direct comparison between simple CWs and CWs with impermeable side walls is not straightforward due to the differentiation between major and minor capture zones with simple CW PRBs. However, it is seen that long (in direction perpendicular to groundwater flow) and narrow CWs are more susceptible to flow by-pass, i.e., the range of deviations of flow direction before flow by-pass begins is smaller. This undermines the typical engineering assumption that a PRB longer than the transverse width of the plume is sufficient for plume intercept and treatment. The presence of funnel arms or VEW increases the maximum permissible deviation of flow direction, while an increase in the hydraulic resistance of the reactor causes the opposite. The latter is also observed for D\&G PRBs in conjunction with the fact that a larger separation distance between drains reduces the susceptibility to flow by-pass.

\section{Acknowledgements}

This research was partially funded by the Austrian Science Fund (FWF) under an Erwin-Schrödinger Fellowship (J2677-N14) and the Florida Water Resources Research Center under a grant from the U.S. Department of Interior (06HQGR0079).

\section{References}

[1] Cunningham, J.A. \& Reinhard, M., Injection-Extraction Treatment Well Pairs: An Alternative to Permeable Reactive Barriers. Ground Water, 40(6), pp. 599-607, 2002. 
[2] Environmental Protection Agency. Capstone Report on the Application, Monitoring, and Performance of Permeable Reactive Barriers for Groundwater Remediation: Volume 1, Performance and Evaluation at Two Sites and Volume 2, Long-Term Monitoring of PRBs: Soil and Groundwater Sampling. EPA/600/R-03/045a and b, 2003.

[3] Starr, R.C. \& Cherry, J.A., In-Situ Remediation of Contaminated Groundwater: The Funnel-and-Gate System. Ground Water, 32(3), pp. 465476, 1994.

[4] Painter, B.D.M., Reactive Barriers: Hydraulic Performance and Design Enhancements. Ground Water, 42(4), pp. 609-617, 2004.

[5] Bundesministerium für Bildung und Forschung. Anwendung von durchströmten Reinigungswänden zur Sanierung von Altlasten. RUBINHandbuch, BMBF-Vorhaben-Nummer 0271241, 2006.

[6] Craig, J.R., Rabideau, A.J. \& Suribhatla R., Analytical Expressions for the Hydraulic Design of Continuous Permeable Reactive Barriers. Advances in Water Resources, 29, pp. 99-111, 2006.

[7] Klammler, H. \& Hatfield, K., Analytical Solutions for Flow Fields near Continuous Wall Reactive Barriers. Journal of Contaminant Hydrology, CONHYD2436 (in press), 2008a.

[8] Klammler, H. \& Hatfield, K., Analytical Solutions for the Flow Fields near Funnel-and-Gate and Drain-and-Gate Reactive Barriers with Hydraulic Losses. Water Resources Research (submitted), 2008b. 